\title{
Up-Regulation of Tight Junction-Related Proteins And Increase of Human Epidermal Keratinocytes Barrier Function by Saccharomycosis Ferment Filtrate
}

\author{
Wen-Rou Wong ${ }^{1}$, Tomohiro Hakozaki ${ }^{2}$, Takashi Yoshii ${ }^{3}$, Tzu-Ya Chen ${ }^{1}$, Jong-Hwei Su. Pang ${ }^{1}$ \\ ${ }^{1}$ Graduate Institute of Clinical Medical Sciences, Chang Gung University, Taiwan; ${ }^{2}$ The Procter \& Gamble Company, Cincinnati, \\ $\mathrm{OH}, \mathrm{USA} .{ }^{3} \mathrm{P} \& \mathrm{G}$ Design Center Godo Kaisha, Kobe, Japan \\ Email: jonghwei@mail.cgu.edu.tw
}

Received March 14 $4^{\text {th }}, 2011$; revised March 25 ${ }^{\text {th }}, 2011$; accepted March $28^{\text {th }}, 2011$.

\begin{abstract}
Saccharomycopsis ferment filtrate (SFF), mainly used in skin care products, has been reported to inhibit inflammatory nitric oxide production and prevent epidermal damage. However, the effects of SFF on epidermal keratinocytes have not yet been explored. We investigated the effects of SFF on skin barrier function using human primary epidermal keratinocytes. Cell viability was determined by MTT assay. The mRNA and protein expression levels of tight junction proteins (claudin-1, -3, -4, occludin, ZO-1) were analyzed by RT-PCR and Western blotting, respectively. The effect of SFF on the barrier formation of epidermal keratinocytes was measured by transepithelial electrical resistance (TER). Rescue of cell death from $\mathrm{H}_{2} \mathrm{O}_{2}$ treatment was evaluated by annexin $V$ staining. SFF, at concentrations that did not cause significant change of cell viability, induced dose-dependent cell-cell adhesion and formation of an organized monolayer structure. Pretreatment of keratinocytes with EGTA, a $\mathrm{Ca}^{2+}$ chelator, did not inhibit the cell-cell adhesion of keratinocytes by SFF, indicating a $\mathrm{Ca}^{2+}$-independent mechanism. The mRNA and protein levels of claudin-1 in keratinocytes were up-regulated by SFF treatment in a dose-dependent manner. The expressions of other tight junctions (TJs) including claudin-3 \& 4, occludin and ZO-1 were also similarly increased in SFF-treated epidermal keratinocytes. The promoting effect of SFF on the barrier function of epidermal keratinocytes was further confirmed by the increased TER value in SFF-treated epidermal keratinocytes. Annexin $V$ staining confirmed that SFF markedly decreased the number of dead cells resulted from $\mathrm{H}_{2} \mathrm{O}_{2}$ injury. Taken together, our results provided the first evidence that SFF enhanced keratinocytes barrier function by increasing the expression of TJs and TER.
\end{abstract}

Keywords: Saccharomycopsis Ferment Filtrate, Keratinocytes, Tight Junctions, Barrier Function, $\mathrm{H}_{2} \mathrm{O}_{2}$ Injury

\section{Introduction}

The epidermis of the skin is the outermost layer of the human body and is in direct contact with the outside world. Epidermal cells bound tightly together provide a physical barrier against injurious chemicals or pathogens from the outside, while simultaneously, preventing water or nutritional loss from the inside of our body. The basis of these effects lies in the well-defined differentiation program of keratinocytes which results in the expression of structure proteins and in turn, function as a protective barrier. Several lines of evidence have indicated that stratum corneum, especially intercellular lipid lamellae and cornified cell envelopes play a central role in the mammalian epidermal barrier [1,2]. Since the study of TJs in skin epidermis is relatively delayed, the presence of TJs, another potential barrier component in human epidermis had been mostly disregarded until the analysis of claudin-1 deficient mice [3]. These claudin-1 deficient mice usually died within 1 day of birth due to defected epidermal barrier function thereby causing excessive transepidermal water loss (TEWL) and severe dehydration [3].

Recent studies have revealed that tight junctions (TJs) are located in a narrow zone of the stratum granulosum, comprising of peripheral and integral proteins that are necessary for connecting neighboring cells and providing 
barrier functions [4-7]. TJs consist of transmembrane proteins including occludins, claudins, and junctional adhesion molecules (JAM), and of plaque proteins, e.g. proteins ZO-1, ZO-2, ZO-3, MUPP-1 and symplekin. A variety of mRNAs coding for $\mathrm{TJ}$ proteins has been identified in adult human skin, including claudin 1, 3, $4,5,7,8,10,12,16$ and 17 , occludin, protein ZO-1, and cingulin [8]. TJ proteins anchored in the membranes of two adjacent cells and interrelated with each other to fasten the cells together as well as prevent other molecules from passing between them. Recent studies enforced the importance the TJs in skin barrier functions [3-6,9-11].

It has been known that yeast extracts are capable of stimulating the growth of other yeast cultures by unknown mechanisms. Studies by Copping and Cook et al in early $20^{\text {th }}$ century, demonstrated that yeast extracts were able to stimulate oxygen uptake of both yeasts and cells of rat liver and abdominal skin; hence the term skin respiratory factors was created [12]. The term Live yeast cell derivative (LYCD) invented by Kaplan et al in 1984 [13] was indeed a synonyms of the skin respiratory factor. LYCD was alcoholic extract from yeast (Saccharomyces cerevisiae), and capable of promoting wound healing, accelerating angiogenesis, reducing the symptoms of burns and cutting wounds, and stimulating epithelization as well as collagen synthesis [12-16]. Saccharomycopsis is a family of yeast which has been used to help fermentation of traditional beverages. Saccharomycopsis Ferment Filtrate (SFF), a type of fermented yeast product, is derived from the filtration of Saccharomycopsis fermented medium. Previous study by Tsai et al demonstrated that in the presence of SFF, the LPS-induced NO production and iNOS protein expression of macrophages were inhibited. Conversely, SFF induced the HO-1 protein expression of macrophages. Furthermore, SFF also exhibited significant protective effect on LPS- or UVR-induced damages in the artificial epidermis [17]. Currently, the topical application of NO synthase inhibitor was shown to accelerate cutaneous barrier recovery after tape stripping in a study using hairless mouse [18]. SFF has been widely used in skin care products for whitening and antiaging. However, there is no scientific evidence concerning its effects on the permeability barrier function of skin. In addition, whether or not the inhibitory effect of SFF on NO production may also exert better barrier function of epidermal keratinocytes is worthy of further elucidation.

In our study, we investigated the effects of SFF on skin barrier function of skin keratinocytes. The induction of TJs including claudin-1, -3, -4, occludin, and ZO-1 were demonstrated. The effect of SFF on increasing transepithelial electrical resistance (TER) and the reduction of apoptosis and $\mathrm{H}_{2} \mathrm{O}_{2}$ damage of culture keratinocytes were also confirmed.

\section{Materials and Methods}

\subsection{Reagents}

Saccharomycopsis Ferment Filtrate (Pitera $\left.{ }^{\circledR}\right)$ was provided by Kobe Technical Center, Procter \& Gamble, Japan.

\subsection{Cell Culture}

Human epidermal keratinocytes were isolated from skins of donors aged 30 - 40 by enzymatic digestion method. Briefly, skin was incubated in $10 \%$ iodine for $30 \mathrm{sec}-$ onds and cleansed with $1 \times \mathrm{PBS}$, then incubated in dispase (type II, $2.5 \mathrm{mg} / \mathrm{ml}$ in $1 \times$ PBS) for 2 hours at $37{ }^{\circ} \mathrm{C}$ to dissociate the epidermis from dermis. The epidermal layer was peeled off carefully and cut into small pieces and incubated in $0.25 \%$ trypsin for 30 minutes at $37{ }^{\circ} \mathrm{C}$ to obtain single keratinocytes. Cells released after enzyme digestion were centrifuged at $1,000 \times \mathrm{g}$ for 10 minutes and the cell pellet was resuspended in keratinocyte serum-free medium (KSFM, GIBCO BRL). Cells in this growth medium were maintained at $37{ }^{\circ} \mathrm{C}$ in a humidified atmosphere of $95 \%$ air and $5 \% \mathrm{CO}_{2}$. Keratinocytes between passages 3 and 5 were used for all the experiments. In the present study, epidermal keratinocytes at $60-70 \%$ confluence were treated with SFF $(0.1-1 \times)$ for 24 hours and used for the analysis of mRNA or protein. All experiments were performed three times in triplicate.

\subsection{MTT Assay}

Epidermal keratinocytes grown in 24-well culture plate with or without SFF treatment for 24 hours were washed once with $1 \times$ PBS, followed by addition of $0.5 \mathrm{ml} \mathrm{KSFM}$ containing $0.05 \mathrm{mg} / \mathrm{ml} \mathrm{3-[4,5-dimethylthiazol-2-yl]-2,}$ 5-diphenyltetrazolium bromide (MTT). After incubation at $37{ }^{\circ} \mathrm{C}$ for one hour, the media was removed and formazan crystals in the cells were solubilized in $1 \mathrm{ml}$ DMSO for OD reading at $570 \mathrm{~nm}$ using a spectrophotometer.

\subsection{RNA isolation and RT-PCR}

Total cellular RNA was isolated by lysis in a guanidinium isothiocyanate buffer followed by single step phenol-chloroform-isoamyl alcohol extraction. Briefly, cells were harvested and lysed in solution D containing $4 \mathrm{M}$ guanidium isothiocyanate, $25 \mathrm{mM}$ sodium citrate $(\mathrm{pH}$ $7.0), 0.5 \%$ sodium sarkosine and $0.1 \mathrm{M} \beta$-merca- 
ptoethanol. Sequentially, $1 / 10$ volume of $2 \mathrm{M}$ sodium acetate ( $\mathrm{pH} 4.0)$, one volume of phenol, and 1/5 volume of chloroform-isoamyl alcohol (49:1, v:v) were added to the homogenate. After vigorous vortexing for 30 seconds, the solution was centrifuged at $10,000 \times \mathrm{g}$ for $15 \mathrm{~min}$ at 4 ${ }^{\circ} \mathrm{C}$. RNA in the aqueous phase was precipitated by the addition of $0.5 \mathrm{ml}$ isopropanol. One $\mu \mathrm{g}$ of total RNA was reverse-transcribed into cDNA by incubating with 200 units of reverse transcriptase in $20 \mu \mathrm{l}$ of reaction buffer containing $0.25 \mu \mathrm{g}$ of random primers and $0.8 \mathrm{mM}$ dNTPs at $42^{\circ} \mathrm{C}$ for one hour. Two $\mu 1$ of the cDNA was used as templates for the PCR reaction. The PCR was performed in a buffer containing $10 \mathrm{mM}$ Tris, $\mathrm{pH} 8.3,50$ $\mathrm{mM} \mathrm{KCl}, 1.5 \mathrm{mM} \mathrm{MgCl}_{2}, 0.2 \mathrm{mM}$ dNTPs, $1 \mu \mathrm{M}$ of each primer, and 5 units Taq DNA polymerase for 30 cycles of denaturation at $94^{\circ} \mathrm{C}$ for $1 \mathrm{~min}$, annealing at $55^{\circ} \mathrm{C}$ for $1 \mathrm{~min}$, and extension at $72^{\circ} \mathrm{C}$ for $2 \mathrm{~min}$. The resulting PCR products were analyzed by $1.5 \%$ agarose gel electrophoresis.

\subsection{Western Blot Analysis}

After SFF treatment, epidermal keratinocytes were rinsed with cold $1 \times$ PBS, scraped and solubilized in lysis buffer (20 mM Tris-Cl, pH 7.5, $150 \mathrm{mM} \mathrm{NaCl}, 1 \mathrm{mM}$ $\mathrm{Na}_{2}$ EDTA, $1 \mathrm{mM}$ Ethylene Glycol-bis ( $\beta$-aminoethylether)-N,N,N',N'-TetraAcetic acid (EGTA), $1 \%$ Triton, $2.5 \mathrm{mM}$ sodium pyrophosphate, $1 \mathrm{mM} \beta$-glycerophosphate, $1 \mathrm{mM} \mathrm{Na} \mathrm{VO}_{4}, 1 \mathrm{mM}$ phenylmethylsulfonyl fluoride, $1 \mu \mathrm{g} / \mathrm{ml}$ aprotinin, and $1 \mu \mathrm{g} / \mathrm{ml}$ leupeptin), followed by centrifugation at $12,000 \times \mathrm{g}$ for 20 minutes at $4{ }^{\circ} \mathrm{C}$. The protein concentrations in the supernatants were determined with a Bradford protein assay kit (Bio-Rad). Cell lysates containing equal amounts of protein were separated by $10 \%$ SDS-PAGE, and transferred onto PVDF membrane (Millipore). The membrane was incubated in blocking solution ( $1 \%$ BSA, $1 \%$ goat serum in 1 $\times$ PBS) for 1 hour, followed by incubation with primary antibody properly diluted in blocking solution. After washing, the membrane was incubated in $1 \times$ PBS containing goat anti-mouse IgG conjugated with horseradish peroxidase (Sigma) for 1 hour. The membrane was washed and the positive signals were developed with enhanced chemiluminescence reagent (Amershan Pharmacia Biotech).

\subsection{TER Measurement}

The TER was measured using the EVOM epithelial voltohmmeter (World Precision Instruments) as described by manufacturer. The TER values were calculated by subtracting the contributions of the bare filter and medium. The TER represents the transepithelial permeabil- ity of the water-soluble ions. A higher value of TER means a lower permeability of ions. The experiment was repeated three times in triplicate.

\section{Results}

\subsection{Cell Viability of Human Skin Keratinocyte in The Presence of SFF In Different Concentrations}

To study the potential effects of SFF on skin epidermal barrier, primary culture of human epidermal keratinocytes were incubated with different concentrations of SFF for 24 hours. SFF at the concentration ranging from 0.1 to $1 \times$ did not cause significant change of cell viability in human epidermal keratinocytes as analyzed by MTT assay (Figure 1A). There was neither cytotoxicity nor proliferative effect of SFF on epidermal keratinocytes. However, the morphological appearance of epidermal keratinocytes as observed by inverted-phase contract microscope showed remarkable changes. After the addition of SFF, epidermal keratinocytes began to adhere to nearby cells and form an organized sheet-like structure. This cell-cell adhesion of epidermal keratinocytes induced by SFF was observed at the concentration of $0.5 \times$ SFF and better organization of the sheet-like structure was observed at the concentration of $1 \times$ SFF (Figure 1B).

\subsection{SFF Induced Sheet-Like Structure of Kera- tinocyte Culture in the Coexistence of EGTA}

The pretreatment of epidermal keratinocytes with $1 \mathrm{mM}$ Ethylene Glycol-bis( $\beta$-aminoethylether)-N,N,N',N'-TetraAcetic acid (EGTA) that chelated the $\mathrm{Ca}^{2+}$ in the culture medium did not affect this SFF-induced re-organization of epidermal keratinocytes in culture, suggesting a $\mathrm{Ca}^{2+}$-indepenent mechanism (Figure 2).

\subsection{SFF Induced Claudin-1 in Cultured Human Keratinocytes}

Since the cell-cell adhesion is likely resulted from the intercellular connection of junction proteins, we then investigated the expressions of tight junction-relatedproteins including claudin-1, claudin-3, claudin-4, occludin, and ZO-1 in SFF-treated epidermal keratinocytes. Epidermal keratinocytes were incubated with different concentration of SFF $(0.1-1 \times)$ for 24 hours and the mRNA and protein levels of claudin-1 were first analyzed. The mRNA and protein levels of claudin-1 in epidermal keratinocyte were both increased in a dose-dependent fashion after the SFF treatment (Figure 3A \& Figure 3B). These levels increased in accordance with the formation of organized sheet-like structure. 


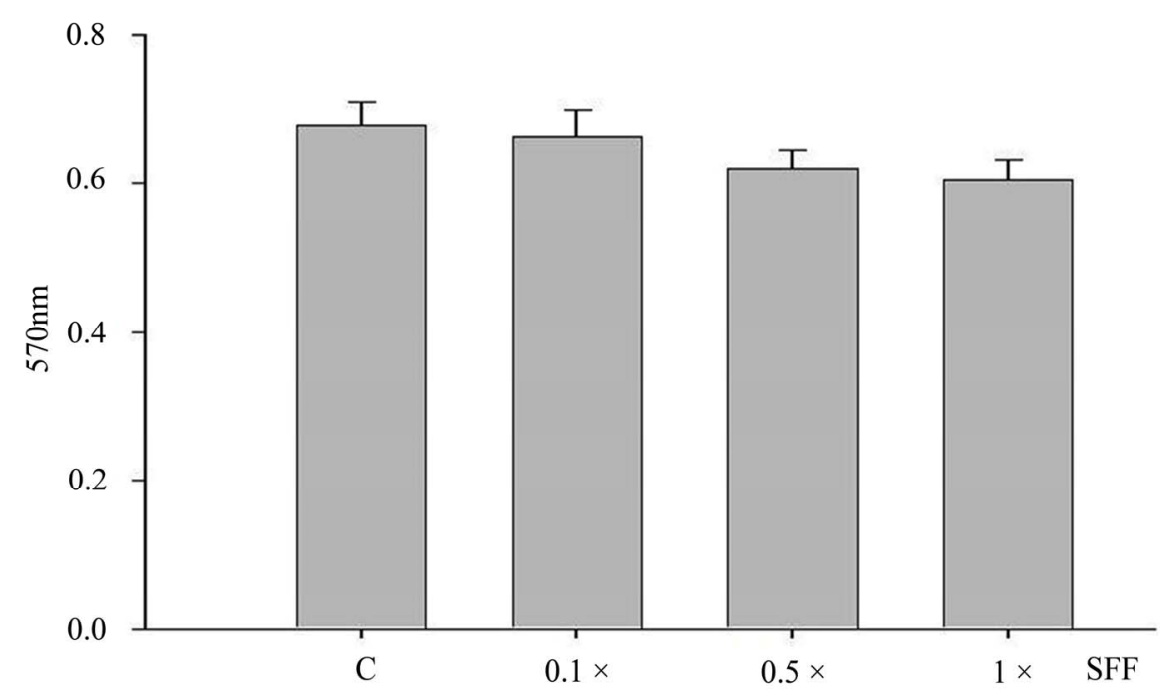

(a)

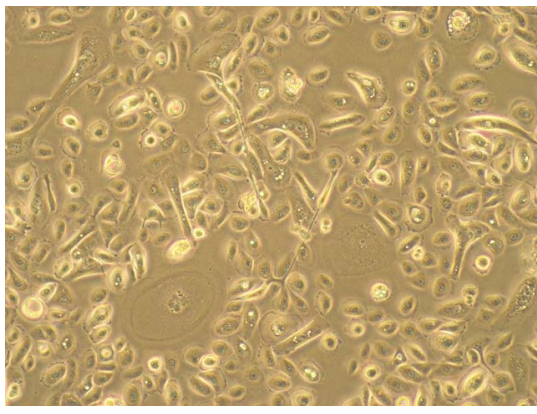

Control

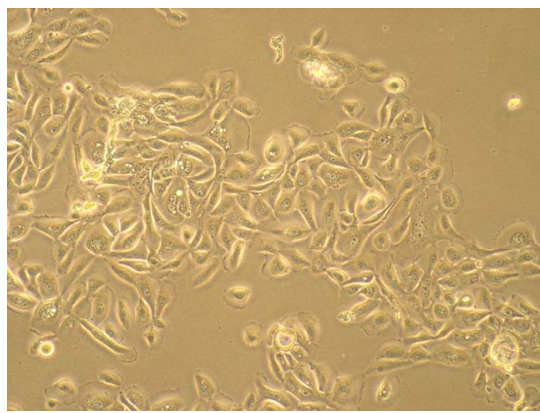

$0.5 \times \mathrm{SFF}$

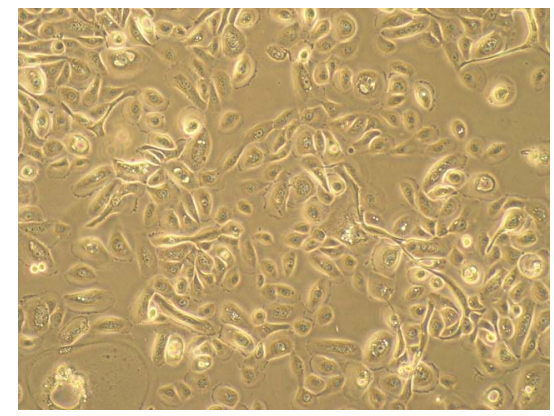

$0.1 \times \mathrm{SFF}$

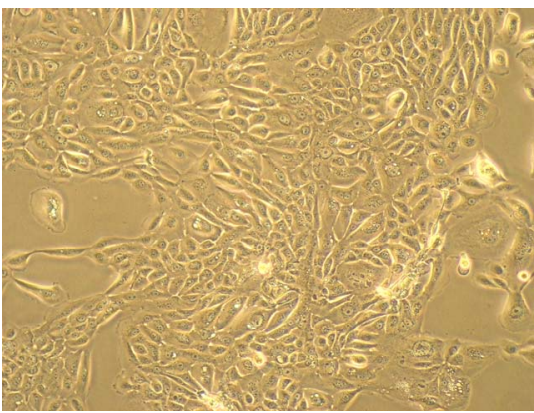

$1 \times \mathrm{SFF}$

(b)

Figure 1. Cell viability and morphological change of epidermal keratinocytes after SFF treatment. Primary epidermal keratinocytes at 60-70\% confluency were treated with different concentration of SFF as indicated and cell viability was measured by MTT assay (A). The morphological change of epidermal keratinocytes was observed by phase contrast inverted microscope and photographed $(200 \times)(B)$. All experiments were done in triplicate.

\subsection{SFF Induced Tight Junction Proteins (Clau- din-3, Claudin-4, Occludin and ZO-1) In Epidermal Keratinocyte}

The expressions of other tight junction-related proteins such as claudin-3, claudin-4, occludin and ZO-1 in epidermal keratinocyte were evaluated by Western blotting. As well as claudin-1, these proteins increased in a dosedependent fashion after the SFF treatment (Figure 4). 


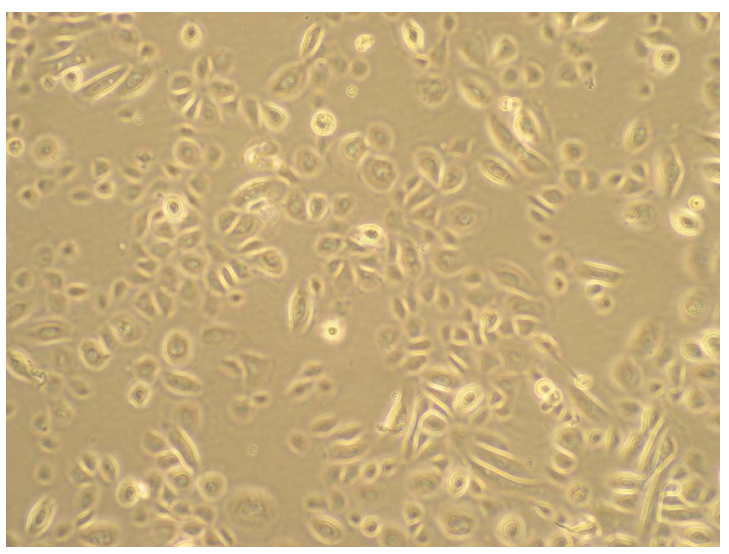

Control

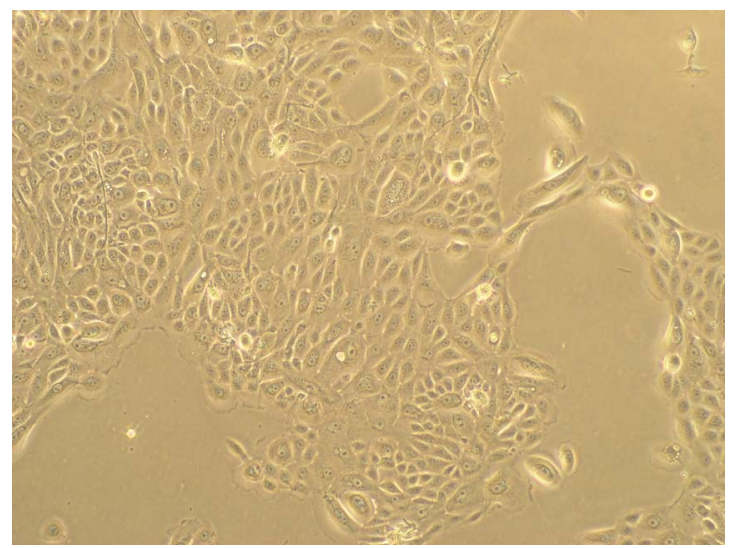

$1 \times \mathrm{SFF}$

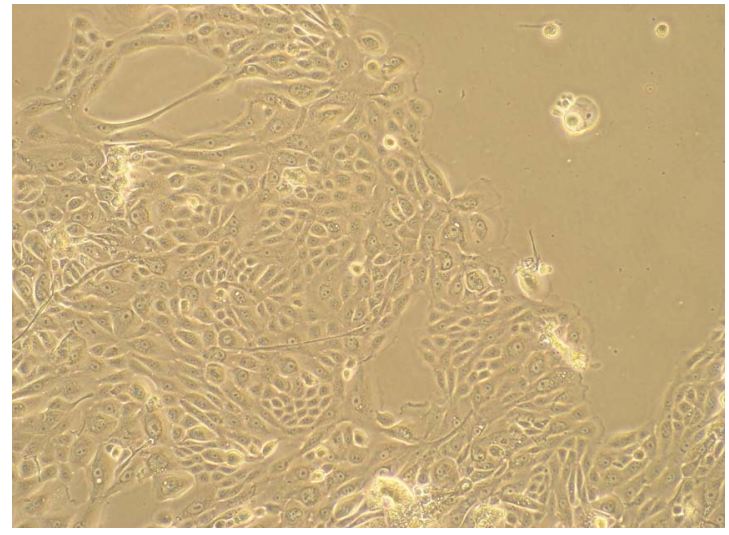

$\mathrm{EGTA}+1 \times \mathrm{SFF}$

Figure 2. Effect of EGTA on the cell-cell adhesion of epidermal keratinocytes induced by SFF or $\mathrm{Ca}^{2+}$. Primary epidermal keratinocytes at 60-70 \% confluency were treated with $1 \times \mathrm{SFF}$ or $1 \mathrm{mM} \mathrm{CaCl}$ in the absence or presence of $1 \mathrm{mM}$ EGTA for $24 \mathrm{hrs}$. The morphological change of epidermal keratinocytes was observed by phase contrast inverted microscope and photographed $(200 \times)$. All experiments were done in triplicate.

\subsection{SFF Elevation of TER in Cultured Human Keratinocytes}

It is known that the expressions of tight junction related-proteins are important determinants of cell permeability and barrier function; we then measured the effect of SFF on TER, a consistent measure of cell permeability. Epidermal keratinocytes were grown on the filter membrane in a transwell chamber and then incubated with SFF (0.1-1×) for 24 hours. The cell-cell adhesion could also be induced in cells grown on a filter membrane. A significant increase in TER with a dose-dependent manner was detected, suggesting a much improved permeability barrier function in SFF-treated epidermal keratinocytes (Figure 5).

\subsection{SFF Inhibition $\mathrm{H}_{2} \mathrm{O}_{2}$-Induced Cell Death in Cultured Keratinocytes}

Whether the increased barrier function as suggested by the increased TER could protect the epidermal keratinocytes from extracellular damage was then tested using hydrogen peroxide $\left(\mathrm{H}_{2} \mathrm{O}_{2}\right) \cdot \mathrm{H}_{2} \mathrm{O}_{2}$ is known to disrupt the tight junction proteins and induce hyperpermeability in epithelial and endothelial cells $[19,20]$. The treatment with $\mathrm{H}_{2} \mathrm{O}_{2}$ induced the shrinkage of epidermal keratinocytes, a morphological indication of apoptosis, with subsequent cell loss. However, in the presence of SFF, the morphology of keratinocyte maintained normal (Figure 6A). Moreover, the cell loss could be reduced by coexistence of SFF as analyzed by MTT assay (Figure 6B).

\section{Discussion}

The understanding of cellular differentiation and formation of intercellular junctions can be demonstrated by keratinocyte culture. Human epidermal keratinocytes stay undifferentiated and do not develop intercellular contacts when cultured in medium containing low $\mathrm{Ca}^{2+}$ $[21,22]$. An increase in extracellular $\mathrm{Ca}^{2+}$ induces the formation of adherens junctions, desmosomes, and then cell stratification [23-25]. $\mathrm{Ca}^{2+}$ also brings about an increase in occludin, claudin-1, and ZO-1 positive cells [26]. $\mathrm{Ca}^{2+}$ activates formation of filopodia, which embed into the neighboring cells. This process is associated with clustering of several TJs at sites of intercellular contacts. Our experiment demonstrated that by adding SFF, keratinocytes migrated to nearby cells resulting in confluent sheets similar to that induced by high concentration of $\mathrm{Ca}^{2+}$. The results were not brought about by keratinocyte proliferation, since MTT analysis of our experiment demonstrated neither cytotoxicity nor proliferative effects on keratinocytes at the tested SFF concentrations. This effect of SFF was not associated with the 


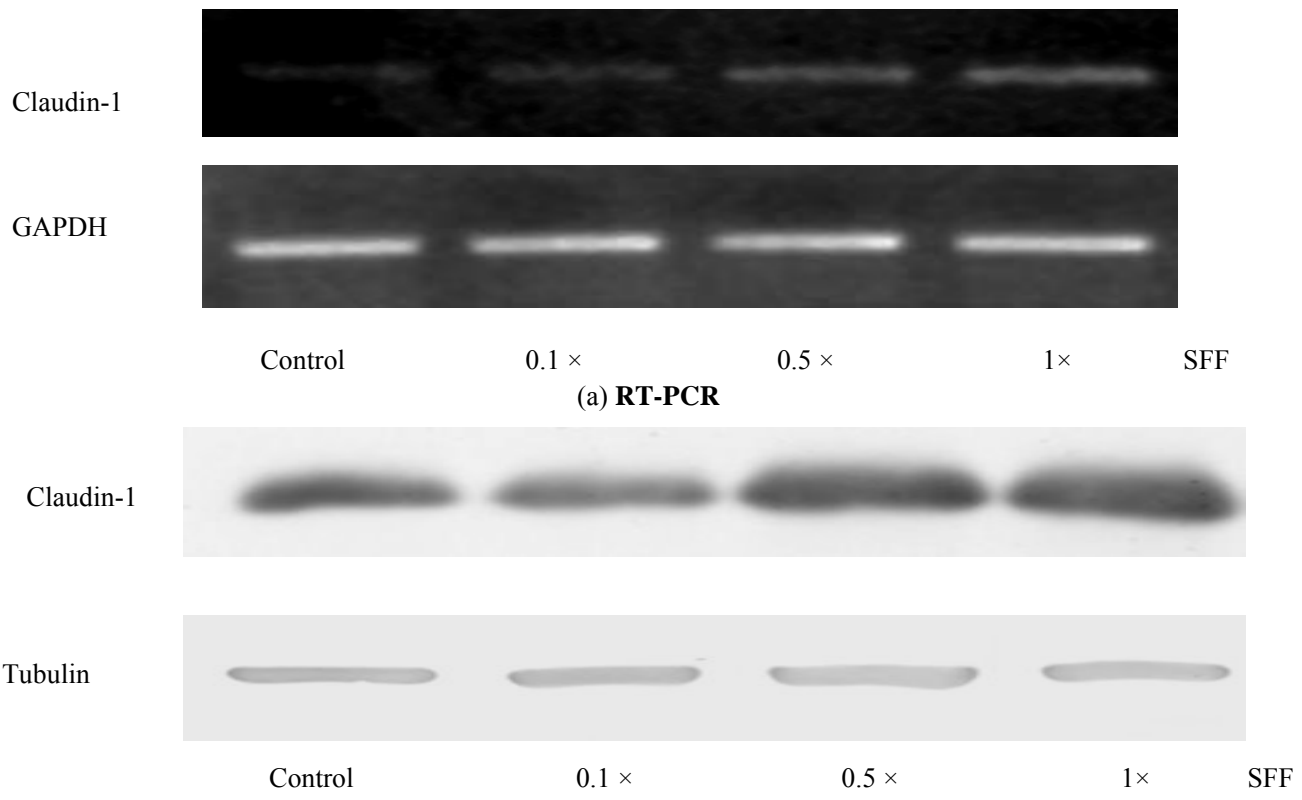

(b) Western Blot

Figure 3. Effect of SFF on the mRNA and protein levels of claudin-1 in epidermal keratinocytes. Primary epidermal keratinocytes at 60-70 \% confluency were treated with different concentration of SFF as indicated. The mRNA and protein levels of claudin-1 in epidermal keratinocytes were analyzed by RT-PCR (A) and Western blot method (B). All experiments were done in triplicate.

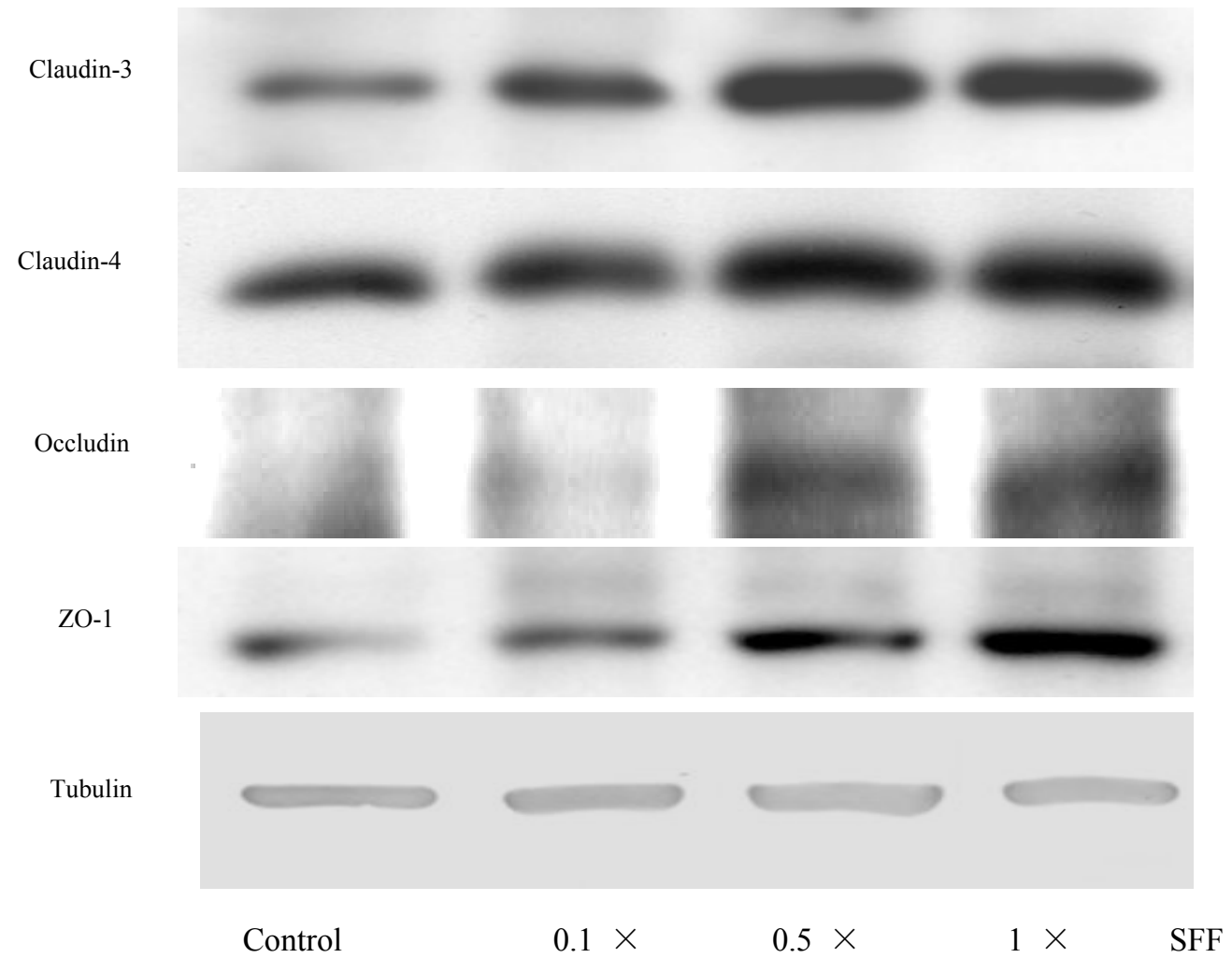

Figure 4. Effect of SFF on the protein levels of TJs in epidermal keratinocytes. Primary epidermal keratinocytes at 60-70 \% confluency were treated with different concentration of SFF as indicated. The protein levels of TJs including claudin-3,-4, occludin and ZO-1 in epidermal keratinocytes were analyzed by Western blot method. All experiments were done in triplicate. 


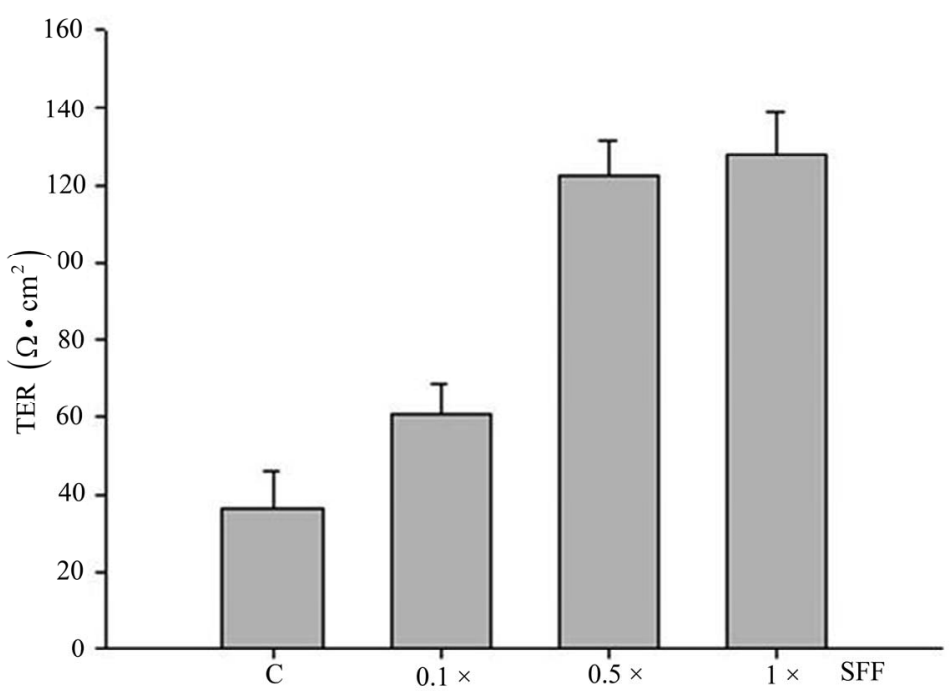

Figure 5. Effect of SFF on TER of epidermal keratinocytes. Primary epidermal keratinocytes were grown on the filter membrane in a transwell chamber and treated with 1× SFF for 24 hrs. TER was then measured by using the EVOM epithelial voltohmmeter. All experiments were done in triplicate.

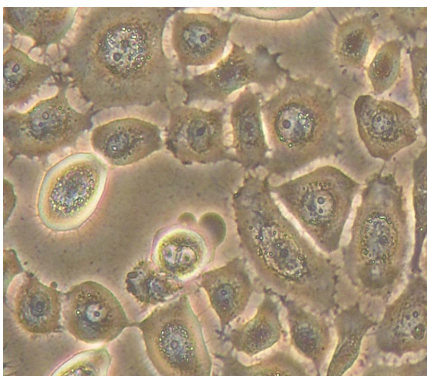

$\mathrm{H}_{2} \mathrm{O}_{2}$

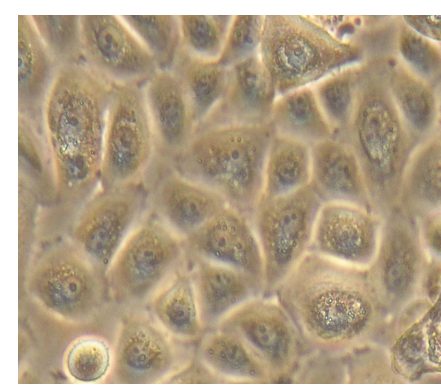

$1 \times \mathrm{SFF}+\mathrm{H}_{2} \mathrm{O}_{2}$

(a)

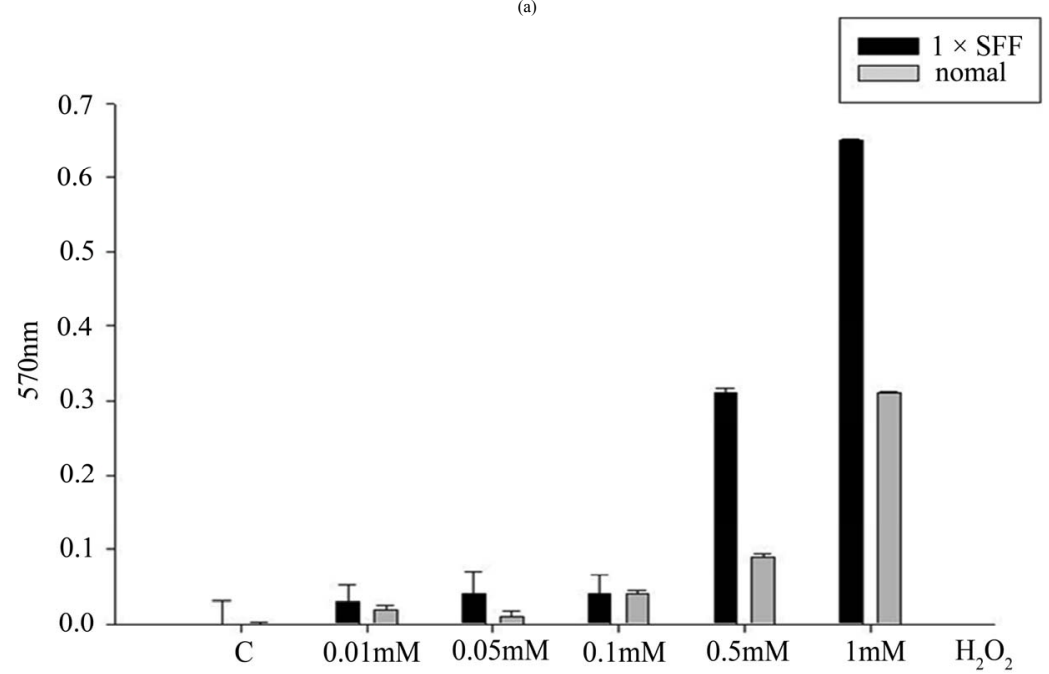

(b)

Figure 6. SFF reduced the $\mathrm{H}_{2} \mathrm{O}_{2}$-induced cell loss in epidermal keratinocytes. Primary epidermal keratinocytes at 60-70\% confluency were treated with $1 \times$ SFF for $24 \mathrm{hrs} . \mathrm{H}_{2} \mathrm{O}_{2}$ at $1 \mathrm{mM}$ was then added to the culture for another $24 \mathrm{hrs}$ and photographed (A). Primary epidermal keratinocytes at 60-70 \% confluency were treated with $1 \times$ SFF for 24 hrs. Different concentration of $\mathrm{H}_{2} \mathrm{O}_{2}$ was then added to the culture for another 24 hrs and cell viability was measured by MTT assay (B). All experiments were done in triplicate. 
presence of $\mathrm{Ca}^{2+}$, since the pretreatment with EGTA, a $\mathrm{Ca}^{2+}$ chelator, did not abolish the cell-cell adhesion of epidermal keratinocytes induced by SFF.

In our experiments, keratinocytes cultured with SFF also increased the TER. The TER of the keratinocyte sheet reflects the transepithelial permeability of water-soluble ions. A higher TER indicates a lower ionic permeability [27]. Meanwhile, the expression of TJs, including claudin-1, $-3,-4$, occludin and ZO-1, were also elevated in a dose-dependent fashion. In stratified epithelia such as human epidermis, the role of TJs was not clearly elucidated from 1970 to the earlier 1980s. The observation of TJs in epidermis by freeze-fracture failed to demonstrate typical belt-like TJ strands surrounded keratinocytes. Only poorly-developed TJ-like strands were rarely encountered [6]. Skin barrier function was initially considered to be provided primarily by the cornified cell envelope, an insoluble complex of cross-linked proteins and lipids [28]. After the anaylsis of claudin-1 deficient mouse, the crucial role of TJs in skin barrier function gained popular interest. Claudin-1 deficient mice died within 1 day of birth and showed severe defects in the permeability barrier of epidermis, despite possessing an intact CCE [3]. Emerging evidence also showed the essential functions of TJs in barrier functions $[4,29]$. Thus the elevated TER and increases in expression of TJs after cultured with SFF, are indicative of an enhancement in skin barrier function.

Exogenous $\mathrm{H}_{2} \mathrm{O}_{2}$ supplementation is known to cause an increased permeability and decreased TER accompanied with disruption of TJs in epithelial cells and endothelial cells [17]. The addition of $\mathrm{H}_{2} \mathrm{O}_{2}$ to epidermal keratinocytes for 24 hours resulted in a dose-dependent cell loss; this effect could be eliminated when cells were simultaneously within the presence of SFF. Annexin V analysis demonstrated less fluorescent staining cells after adding SFF with $\mathrm{H}_{2} \mathrm{O}_{2}$. The results reinforced the protective effect of SFF by preventing apoptosis of keratinocyte from the damage of $\mathrm{H}_{2} \mathrm{O}_{2}$. The enhanced barrier function of epidermal keratinocytes by SFF was confirmed in our study.

The epidermis shows polarization in a broader sense, such that specific layers of the epidermis not only express an unique set of differentiation but also functional markers [30,30,31]. TJs are not only important in the permeability properties of epidermal barriers; they also function in the regulation of keratinocyte proliferation and differentiation. The TJs consist of transmembrane proteins that mediate adhesion, barrier formation, and selective paracellular diffusion of skin. TJs also form a protein network that links the junction to the actin cytoskeleton and recruit different types of signaling pro- teins that regulate junction assembly and function as well as epithelial proliferation and differentiation [32].

In many diseases, alterations in location or quantity of TJs were observed. In hyperkeratotic dermatoses, such as psoriasis vulgaris, lichen planus, and ichthyosis vulgaris, expression of ZO-1 and occludin were relocated to the upper parts of the thickened spinous layer [5]. Electron microscopic studies showed that TJs decreased in number and were attenuated during carcinogenesis, and was associated with loss of tumor differentiation. As such, expression of occludin and ZO-1 is reduced in gastrointestinal adenocarcinoma in relation to histopathological grading. Claudin-1 expression is low or undetectable in breast tumors and breast cancer cell lines, indicating claudin-1 as a possible tumor-suppressor gene [33,34]. Aberrant distribution or decrease expression of occludin, ZO-1, and claudin-1, -4, were observed in Bowen's disease and squamous cell carcinoma of skin [35]. In our experiments, 24 hours after incubation with SFF, the TJs including claudin-1, -3, -4, occludin, and ZO-1 were increased in a dose-dependent fashion, both in transcriptional and translational levels. Skin barrier function deterioration and elevation of TEWL were typical alterations in aged skin [36-38]. Appearance of characteristic wrinkles in claudin-1 deficient mice also points to an important role in TJs in anti-aging [3]. The identification of TJ-associated proteins such as symplekin, which participates in nuclear as well as cytoplasmic polyadenylation, suggests that TJs might contribute to regulation of mRNA stability and localization [39]. These observations indicate that SFF may also contribute to the modulation of keratinocyte differentiation toward a healthier trend and thus improve the quality of photodamaged skin.

Overall, our results demonstrate that SFF can increase skin barrier function by increasing the expression of TJs. This is the first study that elucidates the effects of SFF as an effective moisturizing component. The induction of TJs by SFF may also benefit skin health in the aspects of anti-aging, by modulating keratinocyte differentiation, preventing cellular apoptosis, as well as enhancing skin barrier function. The SFF is a complex mixture, and it is not known which of its many components is responsible for these biological activities. Further studies using fractionations of SFF with functional analysis as well as in vivo study on human skin are warranted for a better understanding of the effects of SFF.

\section{REFERENCES}

[1] N. Kitson and J. L. Thewalt, "Hypothesis: The Epidermal Permeability Barrier is A Porous Medium," Acta Dermato-Venereologica Supplementum, Vol. 208, 2000, pp: 12-15. doi:10.1080/000155500750042808 
[2] P. W. Wertz, "Lipids and Barrier Function of the Skin," Acta Dermato-Venereologica Supplementum, Vol. 208, 2000, pp. 7-11. doi:10.1080/000155500750042790

[3] M. Furuse, M. Hata, K. Furuse, Y. Yoshida, A. Haratake and Y. Sugitani, et al, "Claudin-Based Tight Junctions Are Crucial for The Mammalian Epidermal Barrier: A Lesson From Claudin-1-Deficient Mice," Journal of Cell Biology, Vol. 156, No. 6, 2002, pp. 1099-1111. doi:10.1083/jcb.200110122

[4] M. Malminen, V. Koivukangas, J. Peltonen, S. L. Karvonen, A. Oikarinen and S. Peltonen, "Immunohistological Distribution of The Tight Junction Components ZO-1 and Occludin In Regenerating Human Epidermis," British Journal of Dermatology, Vol. 149, No. 2, 2003, pp. 255-260. doi:10.1046/j.1365-2133.2003.05438.x

[5] K. Pummi, M. Malminen, H. Aho, S. L. Karvonen, J. Peltonen and S. Peltonen, "Epidermal Tight Junctions: Zo-1 and Occludin Are Expressed In Mature, Developing and Affected Skin and In Vitro Differentiating Keratinocytes," Journal of Investigative Dermatology, Vol. 117, No. 5, 2001, pp. 1050-1058. doi:10.1046/j.0022-202x.2001.01493.x

[6] K. Morita and Y. Miyachi, "Tight Junctions in the Skin," Journal of Dermatological Science, Vol. 31, No. 2, 2003, pp. 81-89. doi:10.1016/S0923-1811(03)00038-0

[7] T. Yuki, A. Haratake, H. Koishikawa, K. Morita, Y. Miyachi and S. Inoue, "Tight Junction Proteins in Keratinocytes: Localization and Contribution to Barrier Function," Expiermental Dermatology, Vol. 16, 2007, pp. 324-330. doi:10.1111/j.1600-0625.2006.00539.x

[8] J. M. Brandner, S. Kief, E. Wladykowski, P. Houdek and I. Moll, "Tight Junction Proteins In The Skin," Skin Pharmacology \& Physiology, Vol. 1919, No. 2, pp. 71-77.

[9] D. Tsuruta, K. J. Green, S. Getsios and J. C. Jones, "The Barrier Function of Skin: How to Keep A Tight Lid On Water Loss," Trends in Cell Biology, Vol. 12, No. 8, 2002, pp. 355-357. doi:10.1016/S0962-8924(02)02316-4

[10] G. Bazzoni and E. Dejana, "Keratinocyte Junctions and The Epidermal Barrier: How to Make A Skin-Tight Dress," Journal of Cell Biology, Vol. 156, No. 6, 2002, pp. 947-949. doi:10.1083/jcb.200202116

[11] T. Yuki, A. Haratake, H. Koishikawa, K. Morita, Y. Miyachi and S. Inoue, "Tight Junction Proteins In Keratinocytes: Localization and Contribution to Barrier Function," Expiermental Dermatology, Vol. 16, 2007, pp. 324-330. doi:10.1111/j.1600-0625.2006.00539.x

[12] J. P. Bentley, T. K. Hunt, J. B. Weiss, C. M. Taylor, A. N. Hanson, G. H. Davies, et al, "Peptides from Live Yeast Cell Derivative Stimulate Wound Healing," Archives of Surgery, Vol. 125, No. 5, 1990, pp. 641-646.

[13] J. Z. Kaplan, "Acceleration of Wound Healing by A Live Yeast Cell Derivative," Archives of Surgery, Vol. 119, No. 9, 1984, pp. 1005-1008.

[14] A. J. Dart, B. A. Dowling, C. L. Smith, "Topical treatments in equine wound management," Equine Practice,
Vol. 21, No. 1, 2005, pp. 77-89.

doi:10.1016/j.cveq.2004.11.003

[15] J. M. Liptak, "An Overview of the Topical Management of Wounds," Australian Veterinary Journal, Vol. 75, No. 6, 1997, pp. 408-413. doi:10.1111/j.1751-0813.1997.tb14342.x

[16] M. J. Crowe, R. B. McNeill, D. J. Schlemm, D. G. Greenhalgh and S. J. Keller, "Topical Application of Yeast Extract Accelerates The Wound Healing Of Diabetic Mice," Journal of Burn Care \& Rehabilitation, Vol. 2, 1920, pp. 155-162.

[17] H. H. Tsai, Y. C. Chen, W. R. Lee, C. H. Hu, T. Hakozaki and T. Yoshii, et al, "Inhibition of Inflammatory Nitric Oxide Production and Epidermis Damages by Saccharomycopsis Ferment Filtrate," Journal of Dermatological Science, Vol. 42, No. 3, 2006, pp. 249-257. doi:10.1016/j.jdermsci.2006.01.009

[18] K. Ikeyama, S. Fuziwara and M. Denda, "Topical Application Of Neuronal Nitric Oxide Synthase Inhibitor Accelerates Cutaneous Barrier Recovery and Prevents Epidermal Hyperplasia Induced By Barrier Disruption," Journal of Investigative Dermatology, Vol. 127, No. 7, 2007, pp. 1713-1719.

[19] S. Basuroy, A. Seth, B. Elias, A. P. Naren and R. Rao, "MAPK Interacts With Occludin and Mediates Egf-Induced Prevention of Tight Junction Disruption by Hydrogen Peroxide," Biochemical Journal, Vol. 393, No. 1, 2006, pp. 69-77. doi:10.1042/BJ20050959

[20] H. S. Lee, K. Namkoong, D. H. Kim, K. J. Kim, Y. H. Cheong and S. S. Kim, et al, "Hydrogen Peroxide-Induced Alterations of Tight Junction Proteins In Bovine Brain Microvascular Endothelial Cells," Microvascular Research, Vol. 68, No. 3, 2004, pp. 231-238. doi:10.1016/j.mvr.2004.07.005

[21] J. C. Jones and R. D. Goldman, "Intermediate Filaments and The Initiation of Desmosome Assembly," Journal of Cell Biology, Vol. 101, No. 2, 1985, pp. 506-517. doi:10.1083/jcb.101.2.506

[22] E. J. O'Keefe, R. A. Briggaman and B. Herman, "Calcium-Induced Assembly of Adherens Junctions in Keratinocytes," Journal of Cell Biology, Vol. 105, No. 2, 1987, pp. 807-817. doi:10.1083/jcb.105.2.807

[23] H. Hennings and K. A. Holbrook, "Calcium Regulation of Cell-Cell Contact and Differentiation of Epidermal Cells in Culture. An ultrastructural study," Experimental Cell Research, Vol. 143, No. 1, 1983, pp. 127-142. doi:10.1016/0014-4827(83)90115-5

[24] M. D. Kubler, P. W. Jordan, C. H. O'Neill and F. M. Watt, "Changes In The Abundance and Distribution of Actin and Associated Proteins During Terminal Differentiation of Human Epidermal Keratinocytes," Journal of Cell Science 100, 1991, pp. 153-65.

[25] J. E. Lewis, P. J. Jensen and M. J. Wheelock, "Cadherin Function Is Required for Human Keratinocytes to Assemble Desmosomes and Stratify In Response to Calcium," Journal of Investigative Dermatology, Vol. 102, 
No. 6, 1994, pp. 870-877.

doi:10.1111/1523-1747.ep12382690

[26] K. Pummi, M. Malminen, H. Aho, S. L. Karvonen, J. Peltonen and S. Peltonen, "Epidermal Tight Junctions: Zo-1 and Occludin Are Expressed In Mature, Developing and Affected Skin and In Vitro Differentiating Keratinocytes," Journal of Investigative Dermatology, Vol. 117, No. 5, 2001, pp. 1050-1058. doi:10.1046/j.0022-202x.2001.01493.x

[27] S. A. Francis, J. M. Kelly, J. McCormack, R. A. Rogers, J. Lai and E. E. Schneeberger, et al, "Rapid Reduction of MDCK Cell Cholesterol by Methyl-Beta-Cyclodextrin Alters Steady State Transepithelial Electrical Resistance," European Journal of Cell Biology, Vol. 78, No. 7, 1999, pp. 473-484.

[28] D. Roop, "Defects in the Barrier," Science, Vol. 267, No. 5197, 1995, pp. 474-475. doi:10.1126/science.7529942

[29] T. Yuki, A. Haratake, H. Koishikawa, K. Morita and Y. Miyachi, "Tight Junction Proteins In Keratinocytes: Localization and Contribution to Barrier Function," Experimental Dermatology, Vol. 16, No. 4, 2007, pp. 324-330. doi:10.1111/j.1600-0625.2006.00539.x

[30] F. M. Watt, "Stem cell fate and patterning in mammalian epidermis," Current Opinion in Genetics \& Development, Vol. 11, No. 4, 2001, pp. 410-477. doi:10.1016/S0959-437X(00)00211-2

[31] S. Getsios, A. C. Huen and K. J. Green, "Working Out The Strength And Flexibility of Desmosomes," Nature Reviews Molecular Cell Biology, Vol. 5, No. 4, pp: 271-281. doi:10.1038/nrm1356

[32] K. Matter, S. Aijaz, A. Tsapara and M. S. Balda, "Mammalian Tight Junctions in the Regulation of Epithelial Differentiation and Proliferation," Current Opinion in Cell Biology, Vol. 17, No. 5, 2005, pp. 453-458. doi:10.1016/j.ceb.2005.08.003
[33] F. Kramer, K. White and M. Kubbies, et al, "Genomic Organization of Claudin-1 and Its Assessment in Hereditary and Sporadic Breast Cancer," Human Genetics, Vol. 107, 2007, pp. 249-256.

[34] K. Swisshelm, A. Machl, S. Planitzer, R. Robertson, M. Kubbies and S. Hosier, "SEMP1, A Senescence-Associated cDNA Isolated From Human Mammary Epithelial Cells, Is A Member of An Epithelial Membrane Protein Superfamily," Gene, Vol. 226, No. 2, 1999, pp. 285-295. doi:10.1016/S0378-1119(98)00553-8

[35] K. Morita, S. Tsukita and Y. Miyachi, "Tight JunctionAssociated Proteins (Occludin, Zo-1, Claudin-1, Claudin-4) In Squamous Cell Carcinoma and Bowen's Disease," British Journal of Dermatolog, Vol. 151, No. 2, 2004, pp. 328-334. doi:10.1111/j.1365-2133.2004.06029.x

[36] J. L. Leveque, P. Corcuff, R. J. de and P. Agache, "In Vivo Studies of The Evolution of Physical Properties of the Human Skin with Age," International Journal of Dermatology, Vol. 23, No. 5, 1984, pp. 322-329. doi:10.1111/j.1365-4362.1984.tb04061.x

[37] K. V. Roskos and R. H. Guy, "Assessment of Skin Barrier Function Using Transepidermal Water Loss: Effect of Age," Pharmaceutical Research, Vol. 6, No. 11, 1989, pp: 949-953. doi:10.1023/A:1015941412620

[38] K. P. Wilhelm, A. B. Cua and H. I. Maibach, "Skin aging effect on Transepidermal Water Loss, Stratum Corneum Hydration, Skin Surface Ph and Casual Sebum Content," Archives of Dermatology, Vol. 127, No. 12, 1991, pp. 1806-1809. doi:10.1001/archderm.127.12.1806

[39] D. C. Barnard, K. Ryan, J. L. Manley and J. D. Richter, "Symplekin and xGLD-2 are required for Cpeb-Mediated Cytoplasmic Polyadenylation," Cell, Vol. 119, No. 5, 2004, pp. 641-651. doi:10.1016/j.cell.2004.10.029 